Pacific Journal of Mathematics

ON THE SINGULARITIES OF THE FUNCTION GENERATED
BY THE BERGMAN OPERATOR OF THE SECOND KIND 


\section{ON THE SINGULARITIES OF THE FUNCTION GENERATED BY THE BERGMAN OPERATOR OF THE SECOND KIND}

P. ROSENTHAL

Let $\psi(\lambda, y)=P_{2}(f)$ be Bergman's integral operator of the second kind with domain of definition

$$
W=\left\{(\lambda, y)\left|3^{1 / 2}\right| \lambda \mid<y, \lambda \leqq 0, y>0\right\} \text {. }
$$

Let $f(q)=(q-A)^{-1}, A \in W$. In this paper it is shown that $\psi(\lambda, y)$ has singular points $z=2 A, 2 A(1-w)$, where $w=A^{-1} \lambda$ and $z=\lambda+i y$.

Let

$$
\psi\left(z, z^{*}\right)=P_{2}(f)=\int_{l} E\left(z, z^{*}, t\right) f\left(\frac{z}{2}\left(1-t^{2}\right)\right) \frac{d t}{\sqrt{1-t^{2}}}
$$

be Bergman's integral operator of the second kind. $P_{2}(f)$ maps functions $f$ analytic in one variable in the neighborhood of the origin into solutions of the linear partial differential equation

$$
\psi_{z z^{*}}+N\left(\frac{z+z^{*}}{2}\right)\left(\psi_{z}+\psi_{z^{*}}\right)=0, \quad z=\lambda+i y, \quad z^{*}=\lambda-i y,
$$

$N(\lambda)=-(1 / 12 \lambda)\left(1+b_{1}(-\lambda)^{2 / 3}+\cdots\right)$ is analytic for $-\infty<\lambda<0$ and singular at $\lambda=0 . E\left(z, z^{*}, t\right)$, called the generating function of the operator, is analytic in the three variables $z, z^{*}$, and $t$ providing $\left|z+z^{*}\right|<\left|t^{2} z\right|, l$ is some rectifiable Jordan curve in the upper complex $t$-plane connecting the points -1 and 1, [1], [3].

In a previous paper [7] we obtained some results on the singularities of $P_{2}(f)$ where $f$ is meromorphic and $z, z^{*}$ were treated as independent complx variables. In this paper we let $z^{*}=\bar{z}$ (conjugate of $z$ ) and $N(\lambda)=-1 / 12 \lambda$ (Tricomi case). With these assumptions,

$$
\begin{aligned}
\psi(\lambda, y) & =\int_{-1}^{1} E(u) \frac{f(q)}{\sqrt{1-t^{2}}} d t, \text { where } u=\frac{t^{2} z}{2 \lambda} \\
q & =\frac{1}{2} z\left(1-t^{2}\right) \\
z & =\lambda+i y
\end{aligned}
$$

$E(u)=H(\lambda)\left(F^{(1)}(u)+F^{(2)}(u)\right), \quad F^{(1)}(u)=C_{1} u^{-1 / 6} F_{1}(1 / 6,2 / 3,1 / 3,1 / u)$, $F^{(2)}(u)=C_{2} u^{-5 / 6} F_{2}(5 / 6,4 / 3,5 / 3 ; 1 / u), F_{j}$ is the hypergeometric function $j=1,2, H(\lambda)=C_{3} \lambda^{-1 / 6}, \quad C_{j}$ are constants, $j=1,2,3, \quad(\lambda, y) \in W=$ $\left\{(\lambda, y)\left|3^{1 / 2}\right| \lambda \mid<y, \lambda \leqq 0, y>0\right\}$, 
$l$ (the path of integration $=\left\{t \mid t=e^{i \theta}, 0 \leqq \theta \leqq \pi\right\},[4, \mathrm{p} .107]$

THEOREM. Let $f(q)=(A-q)^{-1}, \quad A=\lambda_{0}+i y_{0} \in W, \lambda / A=w=$ $s+i \sigma, z=\lambda+i y, S_{1}=\left\{(w, z) \mid z=2 A, \pi / 2 \geqq \arg w \geqq \alpha_{1}, \pi / 2>\alpha_{1}>\pi / 3\right.$, $\left.0<\delta_{1} \leqq|w| \leqq 1 / 4-\delta_{2}, 1 / 4>\delta_{1}, \delta_{2}>0,1 / 4>\delta_{1}+\delta_{2}\right\}$,

$S_{2}=\left\{(w, z) \mid z=2 A(1-w)\right.$, same conditions on $w$ as in $\left.S_{1}\right\}$, $S_{3}=\left\{\left(0,2 y_{0}\right)\right\}$. Let $T=S_{1} \cup S_{2} \cup S_{3}$. Then $T$ is a singular set for at least one of the branches of $\psi(w, z)$ defined in (1).

Proof. We consider first the case where $E(u)=H(\lambda) F^{(1)}(u)$.

Domain considerations. (3), (4) imply $\psi(w, z)$ is analytic function of the two complex variables $w, z$ for disc neighborhoods satisfying $0<|w|<1 / 4,|A / 2|<|z|<|A|$, where we have extended $\lambda$ to the complex variable $A w$. Note (1) implies we must specify branch cuts in our definition of $\psi(w, z)$. Since $z=\lambda+i y$ (see (1)), we must also consider the extension of $\lambda, y$ to complex values subject to the above inequalities. Thus we can also obtain nonempty neighborhoods $N_{\tilde{o}}(\lambda)$, $N_{\delta}(y)$ such that $\psi(\lambda, y)$ is an analytic function in $\lambda, y$, where $\lambda, y$ now have been extended to complex values.

In what follows we treat $\psi(w, z)$ as an analytic function in $z$ for fixed $w$.

Consider the function obtained from (1) where we have used the series definition for $F_{1}(u)$,

$$
f(\lambda, y)=\int_{-1}^{1} \sum_{p=0}^{\infty} \frac{a_{p} t^{-2 p}(2 \lambda)^{p}}{z^{p}} \cdot \sum_{p=0}^{\infty}\left(\frac{z}{2 A}\right)^{p}\left(1-t^{2}\right)^{p} t^{-1 / 3} \frac{d t}{\sqrt{1-t^{2}}},
$$

$a_{p}=(\Gamma(p+1 / 6) \Gamma(p+2 / 3) / \Gamma(p+1 / 3) \Gamma(p+1)), \quad \Gamma$ is the Gamma function, $|z|<A,|2 \lambda|<|z|$. From (2) we obtain two series,

$$
\begin{aligned}
& \sum_{p=0}^{\infty}\left(\sum_{k=0}^{\infty} a_{k}\left(\frac{\lambda}{A}\right)^{k} t^{-2 k-1 / 3}\left(1-t^{2}\right)^{p+k-1 / 2}\right)\left(\frac{z}{2 A}\right)^{p} \\
+ & \sum_{p=1}^{\infty}\left(\sum_{k=0}^{\infty} a_{p+k}\left(\frac{\lambda}{A}\right)^{k} t^{-(2(p+k+1 / 6))}\left(1-t^{2}\right)^{k-1 / 2}\left(\frac{2 \lambda}{z}\right)^{p}\right) \\
& |z|<|A|, \quad|2 \lambda|<|z| .
\end{aligned}
$$

We will limit ourselves to the first series in (3) for our analysis of the singularities of $P_{2}(f)$. When $|\lambda| \leqq|A / 2|-\delta,|z| \leqq|A|-\delta$, $|A / 2|>\delta>0$, the operations of summation and integration (with respect to $t$ ) can be interchanged in the first series of (3), our integrals are in the improper Riemann sense. Integrating the first part of (3) by parts, then using the formula, 


$$
\int_{-1}^{1} t^{-1 / 3}\left(1-t^{2}\right)^{\nu+1 / 6} \frac{d t}{\sqrt{1-t^{2}}}=-\frac{1}{2}\left(1-e^{2 \pi i / 3}\right) \frac{\Gamma(1 / 3) \Gamma(\nu+2 / 3)}{\Gamma(\nu+1)},
$$

$[2$, p. 33], we obtain the function

$$
f_{1}(w, z)=\sum_{p=0}^{\infty} \beta_{p}(w)\left(\frac{z}{2 A}\right)^{p},
$$

where

$$
\begin{aligned}
\beta_{p}(w) & =\sum_{k=0}^{\infty} A_{p k} w^{k}, \quad A_{p k}=\frac{\Gamma(k+1 / 6) \Gamma(k+p+1 / 2)}{\Gamma(k+1 / 3) \Gamma(k+1) \Gamma(p+5 / 6)}, \\
w & =\frac{\lambda}{A} .
\end{aligned}
$$

(5) can be rewritten as

$$
\beta_{p}(w)=\alpha_{p} c_{p}(w), \quad \alpha_{p}=\frac{\Gamma(1 / 6) \Gamma(1 / 2+p)}{\Gamma(1 / 3) \Gamma(5 / 6+p)},
$$

$c_{p}(w)=F_{3}(1 / 6,1 / 2+p, 1 / 3 ; w), F_{3}$ a hypergeometric function. Using the asymptotic formula for $F_{3}$ for large $p[6, \mathrm{pp} .235,241$ (23)], we can write $c_{p}(w)$ as

$$
\begin{aligned}
& c_{p}(w)=a_{p}(w) e^{i \pi / 6} R_{1}+a_{p}(w)(1-w)^{-1 / 3-p} R_{2}, \\
& a_{p}(w)=\left(\Gamma\left(\frac{1}{6}\right)\right)^{-1} \Gamma\left(\frac{1}{3}\right)(p w)^{-1 / 6},
\end{aligned}
$$

$p$ sufficiently large, $w \in T_{1}=\left\{w\left|0<\delta_{1} \leqq\right| w \mid \leqq 1 / 4-\delta_{2}, 1 / 4>\delta_{2}\right.$, $\left.\delta_{1}>0,1 / 4>\delta_{1}+\delta_{2}, \pi / 2 \geqq \arg w \geqq \alpha_{1}, \pi / 2>\alpha_{1}>\pi / 3\right\}$,

$$
R_{j}(p, w)=1+R_{0}^{(j)}(p, w),
$$

$\lim _{p \rightarrow \infty} p R_{0}^{(j)}(p, w)=h_{j}(w) \neq 0$ uniformly for $w \in T_{1}, j=1,2$. Using (6), (7), we can rewrite (4) as

$$
\begin{aligned}
f_{1}(w, z) & =\sum_{p=0}^{p=p_{0} \geqq 1} \alpha_{p} c_{p}(w)\left(\frac{z}{2 A}\right)^{p}+\sum_{p=p_{0}+1}^{\infty} c_{1}(p, w) z_{1}^{p}+\sum_{p=p_{0}+1}^{\infty} c_{2}(p, w) z_{2}^{p}, \\
z_{1} & =\frac{z}{2 A}, \quad z_{2}=(1-w)^{-1} \frac{z}{2 A},
\end{aligned}
$$

and

(9) $\quad c_{1}(p, w)=\alpha_{p} a_{p}(w) e^{i \pi / 8} R_{1}, \quad c_{2}(p, w)=\alpha_{p} a_{p}(w)(1-w)^{-1 / 3} R_{2}$,

see (6) for the definition of $\alpha_{p}$, (7) for $a_{p}(w)$.

From (9) we obtain

$$
\rho=\lim _{p \rightarrow \infty}\left|c_{j}(p, w)\right|^{-1 / p}=1
$$


the radius of convergence of the second and third series in (8), and $-\varepsilon<\arg c_{j}(p, w)<\varepsilon, 0<\varepsilon<\pi / 2, p$ sufficiently large, $w \in T_{1}, j=1$, 2.

Proof of (10). From (7) we obtain

$$
1+\varepsilon \geqq\left|R_{j}(p, w)\right| \geqq 1-\varepsilon>0,
$$

$1>\varepsilon>0, p$ sufficiently large, $w \in T_{1}$. So we can take the $p$ th root (say principle branch) of $c_{j}(p, w), j=1,2$, cf (9).

Using the asymptotic formula $(\Gamma(p+A) / \Gamma(p+B)) \sim p^{A-B}$, we conclude the first part of (10). Since $\lim _{p \rightarrow \infty}\left(1+R_{0}^{(j)}(p, w)=1, w \in T_{1}\right.$, see (7), the second part of (10) follows.

$$
z=2 A \quad \text { and } \quad z=2 A(1-w), \quad w \in T_{1},
$$

are singular points of (8).

Proof of (11). (10) satisfies the hypotheses of a theorem of Dienes [5, p. 227]. From this theorem we conclude $z=2 A$ and $z=$ $2 A(1-w)$ are singular points respectively of the second and third series in (8). Further, $c_{j}\left(p=\xi=\rho e^{i \psi}, w\right)$ (see (9)) is an analytic function in $\xi$ in the half-plane $x_{1} \geqq 1, \xi=x_{1}+i y_{1}$, and

$$
\left|c_{j}\left(1+\rho e^{i \psi}, w\right)\right|<e^{\varepsilon \rho}, \varepsilon>0,
$$

and arbitrarily small, $\rho>0$ and sufficiently large, and $-\pi / 2 \leqq \psi \leqq \pi / 2$, $w \in T_{1}, j=1$, 2. This follows from a definition of the remainder term $R_{0}^{(j)}(p, w)$ of $(7)$, see [6, p. 235]. Hence by a theorem of Le Roy and Lindelöf [5, p. 340], we conclude the only possible singular points of the second series in (8) are the points on the ray $\varphi=\varphi_{0}, \varphi_{0}=$ $\arg 2 A$, joining $2 A$ to infinity and the only possible singular points of the third series in (8) are the points on the ray $\phi=\theta_{0}$, $\theta_{0}=\arg 2 A(1-w), w \in T_{1}$, joining $2 A(1-w)$ to infinity. Further, $\arg 2 A \neq \arg (2 A(1-w)), w \in T_{1}$. Hence the singular points $z=2 A$, $z=2 A(1-w), w \in T_{1}$, of the second and third series respectively are not removed upon addition of these two series in (8). This completes the proof of (11).

$$
\left(0,2 y_{0}\right) \text { is a singular point of } \psi(w, z) \text {. }
$$

Proof. Let $w=\lambda / A=\lambda_{0}=0$. (3) then reduces to the first series, and (4) reduces to the hypergeometric function $F_{4}(1,1 / 2,5 / 6$; $\left.\left(y / 2 y_{0}\right)\right)$ times a constant. $F_{4}$ is singular at the point $y=2 y_{0}$, so (12) holds.

From (11), (12) we conclude $T$ is a singular set (see Theorem for the definition of $T)$ of $\psi(w, z)$ for the case $F_{1}$. 
Proof. We note the second series in (3) when integrated with respect to $t$ gives rise to a function $f_{2}(w, z)$ which is regular at the points in $T$.

For the case $F_{2}$ (see (1)) we use the formula

$$
\int_{-1}^{1} t^{-5 / 3}\left(1-t^{2}\right)^{\nu+5 / 6} \frac{d t}{\sqrt{1-t^{2}}}=\frac{1}{2}\left(1-e^{-(2 \pi i / 3)}\right) \frac{\Gamma(-1 / 3) \Gamma(\nu+4 / 3)}{\Gamma(\nu+1)}
$$

[2, p. 33].

Proceeding as above, we then conclude $T-\left\{\left(0,2 y_{0}\right)\right\}$ is a singular set for the case $F_{2}$. (1) thus can be written as the sum of two functions,

$$
\psi(w, z)=\frac{1}{z^{5 / 6}}\left(g(w, z)=z^{2 / 3} P_{1}(w, z)+P_{2}(w, z)\right),
$$

where $P_{j}$ is singular at the points in $T-\left\{\left(0,2 y_{0}\right)\right\}, j=1,2$. This follows from the linearity of the operator $P_{2}(f)$.

$$
\text { At least one of the branches of } g(w, z) \text { of (15) is }
$$$$
\text { singular for points in } T-\left\{\left(0,2 y_{0}\right)\right\} \text {. }
$$

Proof of (14). $z^{2 / 3}$ can be one of the three branches,

$$
\alpha_{1}=R^{2 / 3} e^{i 2 / 3 \theta}, \quad \alpha_{2}=R^{2 / 3} e^{i(2 / 3 \theta+2 / 3 \pi)}, \quad \alpha_{3}=R^{2 / 3} e^{i(2 / 3 \theta+4 / 3 \pi)}, \pi>\theta>-\pi .
$$

We form the sum

$$
\sum_{i=1}^{3} g_{i}(w, z)=\sum_{i=1}^{3} \alpha_{i} P_{1}(w, z)+3 P_{2}(w, z) \text {. }
$$

We note $\sum_{i=1}^{3} \alpha_{i} P_{1}(w, z)=0,|w|<1 / 4 ;|A / 2|<|z|<|A|$ (see (3)). So if all the branches of $\psi(w, z)$ in (13) were regular at the points in $T-\left\{\left(0,2 y_{0}\right)\right\}$, then $P_{2}(w, z)$ would be regular at the same points, a contradiction. For $w=\lambda / A=\lambda_{0}=0, P_{2}(0, z)=0$, hence $\left(0,2 y_{0}\right)$ is a singular point for all branches (13) (see (12)). This completes the proof of our Theorem.

\section{REFERENCES}

1. S. Bergman, Two-dimensional transonic flow patterns, Amer. J. Math., 70 (1948), 856-891.

2. - On an initial value problem in the theory of two-dimensional transonic flow patterns, Pacific J. Math., 32 (1970), 29-46.

3. S. Bergman and R. Bojanic, Application of integral operators to the theory of partial differential equations with singular coefficients, Arch. Rational Mech. Anal., 4 (1962), $323-340$.

4. - Integral Operators in the Theory of Linear Partial Differential Equations, Springer-Verlag, Band 23, 3rd ed., 1971. 
5. P. Dienes, The Taylor Series, Oxford University Press, 1931.

6. Luke Yudell, The Special Functions and Their Approximation, Vol. 1, Academic Press, 1969.

7. P. Rosenthal, On the Bergman integral operator for an elliptic partial differential equation with a singular coefficient, Pacific J. Math., 35 (1970), 493-497.

Received June 16, 1972. This work was supported in part by NSF Grant DP-33779 at Stanford University.

STANFORD UNIVERSITY 


\section{PACIFIC JOURNAL OF MATHEMATICS}

\section{EDITORS}

RICHARD ARENS (Managing Editor)

University of California

Los Angeles, California 90024

R. A. Beaumont

University of Washington

Seattle, Washington 98105
J. DUGUNDJI*

Department of Mathematics

University of Southern California

Los Angeles, California 90007

D. Gilbarg and J. Milgram

Stanford University

Stanford, California 94305

\section{ASSOCIATE EDITORS}
E. F. BECKENBACH
B. H. NeumanN
F. WOLF
K. YosHIDA

\section{SUPPORTING INSTITUTIONS}

UNIVERSITY OF BRITISH COLUMBIA
CALIFORNIA INSTITUTE OF TECHNOLOGY
UNIVERSITY OF CALIFORNIA
MONTANA STATE UNIVERSITY
UNIVERSITY OF NEVADA
NEW MEXICO STATE UNIVERSITY
OREGON STATE UNIVERSITY
UNIVERSITY OF OREGON
OSAKA UNIVERSITY

UNIVERSITY OF BRITISH COLUMBIA

UNIVERSITY OF CALIFORNIA

MONTANA STATE UNIVERSITY

UNIVERSITY OF NEVADA

OREGON STATE UNIVERSITY

OSAKA UNIVERSITY
UNIVERSITY OF SOUTHERN CALIFORNIA

STANFORD UNIVERSITY

UNIVERSITY OF TOKYO

UNIVERSITY OF UTAH

WASHINGTON STATE UNIVERSITY

UNIVERSITY OF WASHINGTON

\section{AMERICAN MATHEMATICAL SOCIETY} NAVAL WEAPONS CENTER

* C. R. DePrima California Institute of Technology, Pasadena, CA 91109, will replace J. Dugundji until August 1974. 


\section{Pacific Journal of Mathematics}

\section{Vol. 48, No. $1 \quad$ March, 1973}

Jan Aarts and David John Lutzer, Pseudo-completeness and the product of Baire

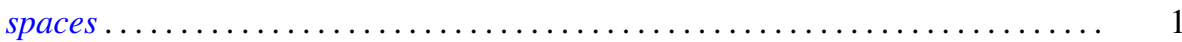

Gordon Owen Berg, Metric characterizations of Euclidean spaces ............ 11

Ajit Kaur Chilana, The space of bounded sequences with the mixed topology ..... . 29

Philip Throop Church and James Timourian, Differentiable open maps of

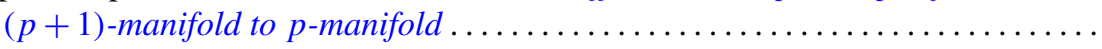

P. D. T. A. Elliott, On additive functions whose limiting distributions possess a finite

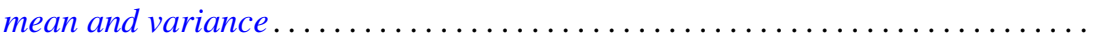

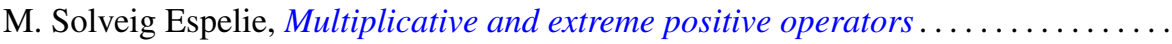

Jacques A. Ferland, Domains of negativity and application to generalized convexity

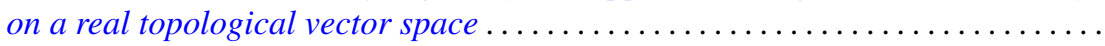

Michael Benton Freeman and Reese Harvey, A compact set that is locally holomorphically convex but not holomorphically convex ...............

Roe William Goodman, Positive-definite distributions and intertwining

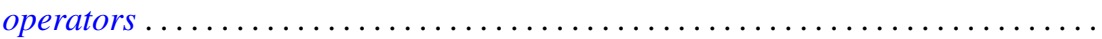

Elliot Charles Gootman, The type of some $C^{*}$ and $W^{*}$-algebras associated with

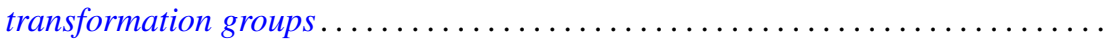

David Charles Haddad, Angular limits of locally finitely valent holomorphic

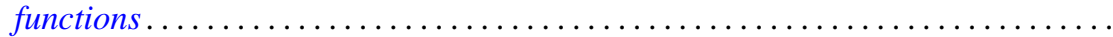

William Buhmann Johnson, On quasi-complements .

William M. Kantor, On 2-transitive collineation groups of finite projective spaces...

Joachim Lambek and Gerhard O. Michler, Completions and classical localizations of right Noetherian rings

Kenneth Lamar Lange, Borel sets of probability measures ......

David Lowell Lovelady, Product integrals for an ordinary differential equation in a Banach space

Jorge Martinez, A hom-functor for lattice-ordered groups .........

W. K. Mason, Weakly almost periodic homeomorphisms of the two sphere ....

Anthony G. Mucci, Limits for martingale-like sequences .......

Eugene Michael Norris, Relationally induced semigroups ...

Arthur E. Olson, A comparison of c-density and $k$-density ......

Donald Steven Passman, On the semisimplicity of group rings of linear groups.

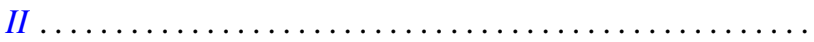

Charles Radin, Ergodicity in von Neumann algebras .

P. Rosenthal, On the singularities of the function generated by the Bergman operator of the second kind.

Arthur Argyle Sagle and J. R. Schumi, Multiplications on homogeneous spaces,

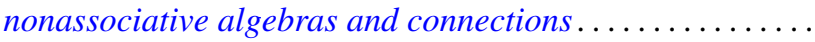

Leo Sario and Cecilia Wang, Existence of Dirichlet finite biharmonic functions on

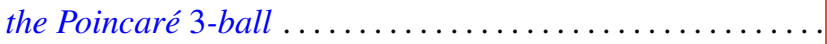

Ramachandran Subramanian, On a generalization of martingales due to Blake ..

Bui An Ton, On strongly nonlinear elliptic variational inequalities.

Seth Warner, A topological characterization of complete, discretely valued

fields. 